\title{
Biomarkers and focused ultrasound: the future of liquid biopsy for brain tumor patients
}

\author{
Jordina Rincon-Torroella ${ }^{1}$ (D) Harmon Khela ${ }^{1} \cdot$ Anya Bettegowda $^{1} \cdot$ Chetan Bettegowda $^{1}$
}

Received: 23 August 2021 / Accepted: 28 August 2021 / Published online: 6 October 2021

(c) The Author(s) 2021

\begin{abstract}
Introduction Despite advances in modern medicine, brain tumor patients are still monitored purely by clinical evaluation and imaging. Traditionally, invasive strategies such as open or stereotactic biopsies have been used to confirm the etiology of clinical and imaging changes. Liquid biopsies can enable physicians to noninvasively analyze the evolution of a tumor and a patient's response to specific treatments. However, as a consequence of biology and the current limitations in detection methods, no blood or cerebrospinal fluid (CSF) brain tumor-derived biomarkers are used in routine clinical practice. Enhancing the presence of tumor biomarkers in blood and CSF via brain-blood barrier (BBB) disruption with MRI-guided focused ultrasound (MRgFUS) is a very compelling strategy for future management of brain tumor patients.

Methods A literature review on MRgFUS-enabled brain tumor liquid biopsy was performed using Medline/Pubmed databases and clinical trial registries.

Results The therapeutic applications of MRgFUS to target brain tumors have been under intense investigation. At highintensity, MRgFUS can ablate brain tumors and target tissues, which needs to be balanced with the increased risk for damage to surrounding normal structures. At lower-intensity and pulsed-frequency, MRgFUS may be able to disrupt the BBB transiently. Thus, while facilitating intratumoral or parenchymal access to standard or novel therapeutics, BBB disruption with MRgFUS has opened the possibility of enhanced detection of brain tumor-derived biomarkers.

Conclusions In this review, we describe the concept of MRgFUS-enabled brain tumor liquid biopsy and present the available preclinical evidence, ongoing clinical trials, limitations, and future directions of this application.
\end{abstract}

Keywords Biomarkers · Focused Ultrasound · Gliomas · Liquid biopsy · Review

\section{Introduction}

From 2013 to 2017, the average annual age-adjusted incidence rate of all central nervous system (CNS) tumors in the United States was 23.79 per 100,000 people [1]. Glioblastoma (GB) represents the most common malignant primary brain tumor, with a dismal five-year survival rate of less than 7 percent $[1,2]$. Therapeutic approaches for primary brain tumors are typically multimodal, with the current standard of care of GB involving maximal surgical resection followed by the Stupp protocol: chemotherapy with temozolomide (TMZ) and concomitant radiation therapy [3-6].

Chetan Bettegowda

cbetteg1@jhmi.edu

1 Department of Neurosurgery, Johns Hopkins University School of Medicine, $600 \mathrm{~N}$ Wolfe St, Phipps 118, Baltimore, MD 21128, USA
Yet, despite extensive research efforts, many patients still experience poor outcomes and low survival rates. Due to the diffusely infiltrative nature of brain tumors, gross total resection is often not curative but rather a measure to increase survival, alleviate neurological symptoms, and optimize the patient's ability to tolerate subsequent post-surgical treatment $[3,4]$. Profound genetic, epigenetic, and morphological heterogeneity are hallmarks of primary brain tumors. Such heterogeneity evolves over time as these tumors adapt to treatment, hindering complete tumor eradication and promoting treatment-resistant recurrences. The radiographic and clinical similarities between tumor recurrence and pseudoprogression complicate the management of brain tumor patients who too often require repeat surgeries to guide care [7]. Liquid biopsy is still constrained by the evolving understanding of tumor biomarkers and their limited representation in body fluids compared to normal tissue elements. Thus, enhancing the presence of tumor biomarkers in blood 
and cerebrospinal fluid (CSF) via brain-blood barrier (BBB) disruption with MRI-guided focused ultrasound (MRgFUS) is a very compelling strategy for future management of brain tumor patients. The present review describes the current research, applications, challenges, and limitations of combining MRgFUS with brain tumor biomarkers.

\section{The challenges of brain tumor therapy}

Poor prognoses for brain tumor patients can be partially attributed to the inadequate penetration of chemotherapeutic agents into the CNS due to the selective permeability of the BBB [8].

Functionally, the BBB comprises endothelial cells, astrocyte end-feet, and pericytes, all of which are bound together via tight junctions that prevent the diffusion of most substances into the brain [9]. Transport across the BBB is typically achieved through passive diffusion of small (less than 400 daltons), non-polar lipophilic molecules or via active transport [10]. Due to this highly selective permeability, delivery and penetration of most brain tumor drugs or smallmolecule therapeutics is often severely limited. Thus, nearly $98 \%$ of small-molecule drugs do not pass through the BBB $[8,11]$. In primary brain malignancies, the $\mathrm{BBB}$ is dysfunctional, and patients have variable regions of $\mathrm{BBB}$ disruption, indicating abundant spatial diversity within different areas of the tumor itself [12]. The intertumor and intratumor heterogeneity plagues molecular-based brain tumor therapies and makes it difficult to ascertain the penetration and efficacy of therapeutic agents. Although most chemotherapeutic agents feature large molecular weights, TMZ is a smaller, lipophilic molecule (194 daltons). This characteristic facilitates its transport across the BBB and its ability to reach therapeutic threshold concentrations in the CSF [13]. However, due to the limited impact of TMZ on patient survival and the likelihood of TMZ-related resistance, novel delivery systems are increasingly being developed to improve brain tumor treatments [14].

\section{Enhancing the delivery of therapies}

Potential solutions to bypassing these limitations imposed by the BBB have included transiently disrupting the BBB or improving drug delivery into brain tumors using nanoparticle/microparticle drug carriers, peptide-based drug delivery, radiotherapy, or local delivery [12]. Another approach includes using convection-enhanced delivery, a therapeutic strategy that slowly coordinates targeted delivery of drugs into the brain using surgically placed small-diameter catheters to infuse the target site and reduce distant spread [15]. Yet, the disadvantages of these various methods have precluded successful implementation in patients. Direct intracranial injection and convection-enhanced delivery are limited by the risk of surgical implantation and the difficulty in executing repeated deliveries [16]. Radiotherapy, while potentially overcoming challenges imposed by the BBB, can have variable efficacy, hamper the immune system, and damage healthy brain tissue. Chemically altering the BBB to improve disruption can lead to unforeseen off-target effects and risk healthy brain tissue [17]. Modifying therapeutics themselves can have low spatial specificity or even result in off-target effects [18]. These existing limitations, together with safety and efficacy concerns, hamper the routine use of those interventions. As a result, noninvasive strategies are becoming more appealing to implement transient and safe modifications to the BBB and improve permeability. MRgFUS has emerged as a valuable intervention for overcoming the anatomical impediments posed by the BBB given the precision afforded by concurrent MRI. To date, studies across various preclinical brain tumor models have exhibited promising results to improve access to brain tumor tissue and enhance systemic treatment efficacy [19-30].

\section{The use and limitations of brain tumor biomarkers}

Beyond limiting therapeutic access into the brain, the low permeability of the BBB additionally hinders the release of biomarkers of brain pathology into the peripheral circulation [31]. Such molecular biomarkers can include DNA mutations, epigenetic alterations, DNA copy number alterations, protein, or microRNA expression, among others. Biomarker detection in brain tumors can be crucial in providing information on the diagnosis, prognosis, and development of individualized treatment for patients harboring targetable molecular changes, which has led to the integration of molecular marker detection in clinical practice [32]. Traditional approaches to detecting tumor-derived biomarkers include direct surgical tissue biopsy to analyze the tumor landscape. However, repeated biopsies may not be feasible due to the elevated risk of complications and morbidity for brain tumor patients. Although surgical resection or stereotactic biopsies are performed for histologic confirmation and genetic profiling, these techniques require brain surgery. Repeated tumor biopsies may be needed to track tumor development or treatment amenability and can be accompanied by severe complications such as hemorrhage or infection [33]. Stereotactic biopsy for brain tumor diagnosis carries a 5-10\% risk of significant morbidity and substantial complications, as studied in meta-analyses and populationlevel studies [33, 34]. Therefore, repeated tumor biopsies to track treatment response, change in tumor biology, and distinguishing between recurrence and pseudoprogression are not often feasible. Noninvasive liquid biopsies are now emerging as a way to provide the same information that traditional tumor biopsies offer but in a safer modality [35, 36]. Through liquid biopsies, brain tumor biomarkers can 
be detected in peripheral blood or CSF (Table 1) [37-67]. This way, the evolution of a tumor and a patient's treatment response could be tracked through a simple, minimally invasive test.

\section{The evolution of liquid biopsy and biomarker detection in cancer}

Liquid biopsy refers to detecting components in human fluids that are pathologically derived (e.g., tumor-derived). Common analytes of liquid biopsies for cancer include circulating tumor cells (CTC), cell-free DNA (cfDNA), and circulating tumor DNA (ctDNA) [68]. ctDNA is DNA detectable in circulation thought to be released during tumor cell apoptosis or necrosis [44]. Contrarily, cfDNA may include both germline and ctDNA. The first report of CTC is attributed to Thomas Ashworth in 1869, who described tumoral cells in the blood of a patient with metastases (Fig. 1) [69]. It was not until 1948 that Mandel et al. reported the presence of cfDNA as non-cell-bound nucleic acids in the blood of cancer patients [70]. But, it would not be until 1994 that ctDNA was distinguished from cfDNA by proving the presence of cancer-specific mutations [71, 72]. Likewise, reliable ctDNA measurements still had to wait until the late 2000s [73]. And detection is still a significant challenge: one $\mathrm{mL}$ blood sample contains a diminutive amount of cfDNA, of which sometimes less than $0.01 \%$ is ctDNA, especially in early cancer stages [74].

Although ctDNA and cfDNA are the most common targets of liquid biopsies, other studied analytes include proteins, mRNA, miRNA, or extracellular microvesicles [68]. Each of those analytes is present in a minimal quantity in human blood or CSF. Consequently, highly sensitive and specific extraction and amplification methods are necessary to make liquid biopsy a reality. Over the past decade, the incredible technical evolution has led to several clinical trials where ctDNA detection showed a correlation with response to treatment or recurrence in non-CNS cancers. Thus, it is already becoming an effective surveillance and follow-up technique in tumors such as breast and lung cancer $[75,76]$. The same application in brain malignancies is constrained by specific limitations related to the nature and localization of CNS tumors.

Several publications have shown the possibility of detecting circulating brain tumor biomarkers [37-67]. A summary can be found in Table 1. Even though the BBB is somewhat disrupted in higher grade gliomas, it remains a prominent obstacle to tumor biomarker release into the bloodstream [77]. In fact, ctDNA is detectable in less than $10 \%$ of patients with gliomas compared to $>75 \%$ of patients with other solid tumors, including advanced pancreatic, ovarian, colorectal, bladder, gastroesophageal, breast, melanoma, hepatocellular, and head and neck cancers [44]. These limitations point to an urgent clinical need to explore alternative approaches that can noninvasively enhance the release of brain tumorderived biomarkers into the peripheral circulation. The intersection of low-intensity pulsed MRgFUS and liquid biopsies has emerged as a means to improve access to brain tumor biomarkers by inducing transient BBB opening [78-81].

\section{The role of focused ultrasound in brain tumors}

Focused ultrasound has been established as a BBB disruption technique to ablate tissue or enhance drug delivery. High-intensity focused ultrasound (HIFU) is used for brain tumor ablation by significantly increasing tissue temperatures using ultrasound energy [82, 83]. This energy is passed through and distributed over the skull. The precise targeting of this energy to the tumor tissue is accomplished via stereotactic, steerable systems like MRgFUS [84, 85]. On the other hand, low-intensity pulsed ultrasounds are a potentially safer and quicker method in which a lower intensity of ultrasound energy is used to prevent tissue damage. Biologically, low-intensity FUS increases the permeability of the BBB through the strategic use of intravenous microbubbles [82]. The ultrasound waves cause a physical cavitation effect in the microbubbles when those pass through the FUS target region [82]. The microbubbles expand until inertial cavitation and eventually collapse, thereby mechanically disrupting the BBB via cellular membrane perforation or blood-tissue permeation [86, 87]. Nevertheless, despite such promising effects, FUS must be precisely controlled. Parameters such as the exposure frequency, microbubble diameter, pulse-repetition frequency, and burst duration can impact the extent of BBB disruption and the penetration of therapeutic agents into the brain [87]. Therefore, lowintensity FUS combined with microbubbles has emerged as an attractive approach to safely and focally induce mechanical disruptions in the BBB to improve therapeutic molecule delivery to the brain. Ongoing preclinical trials for various neurological impairments, including brain cancers [88], Alzheimer's disease [89], and Parkinson's disease [90], have set the foundation for the clinical translation of FUS. Human clinical trials are also determining the feasibility of this technique in various patient groups [91-94]. For example, Lipsman and colleagues demonstrated BBB opening without adverse effects after microbubble treatment with MRgFUS in 5 patients with Alzheimer's [91]. Mainprize and colleagues tested the use of MRgFUS with concomitant TMZ or liposomal doxorubicin in 5 high-grade glioma patients [92]. Both groups considered gadolinium enhancement at the sonication site as a confirmation of BBB opening. The new area of enhancement in MRI resolved overtime (24 h), indicating the reversibility of the BBB disruption [91, 92]. Employing MRgFUS to disrupt the BBB and thereby facilitate the release of brain tumor biomarkers may be an 


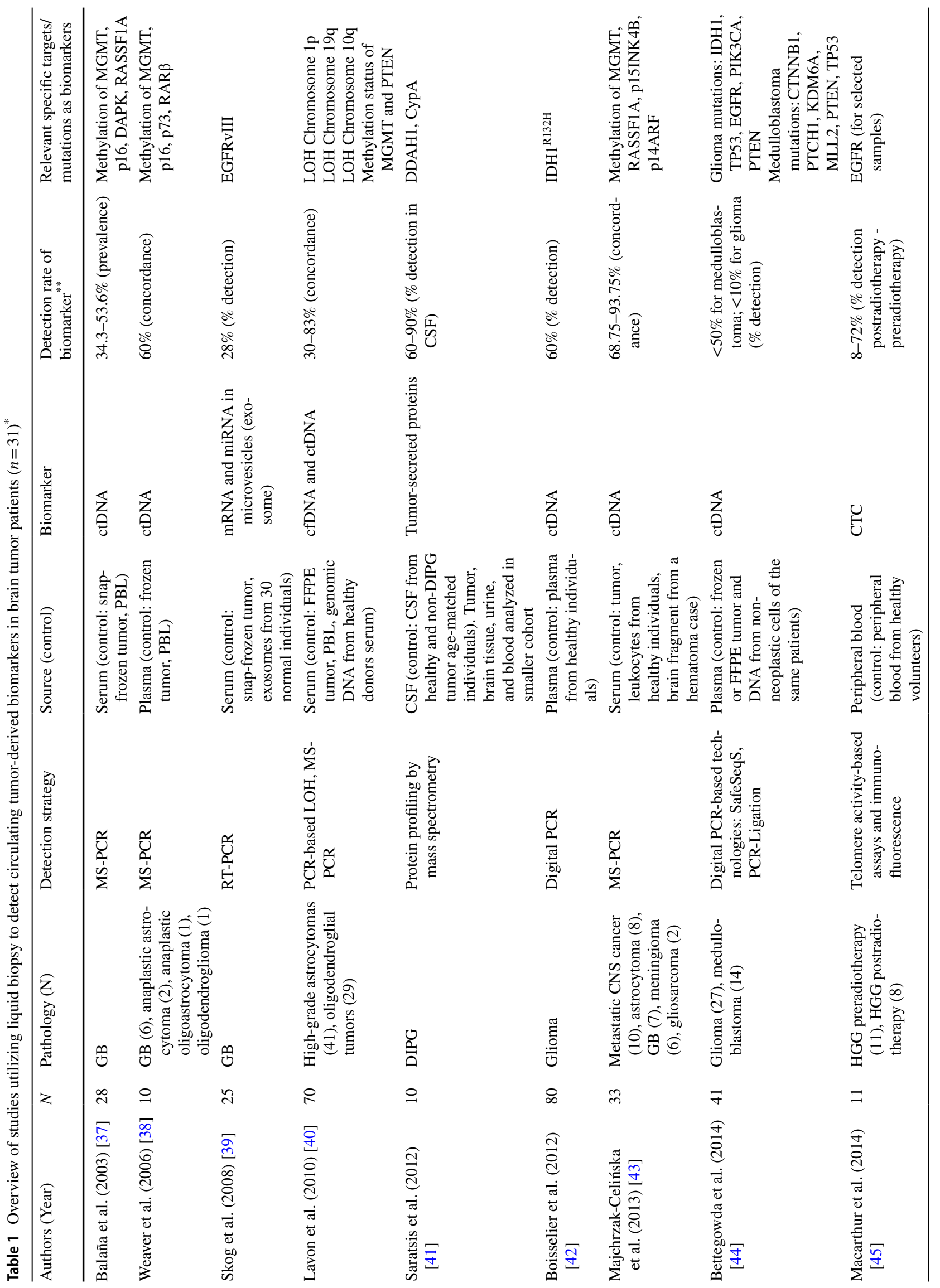




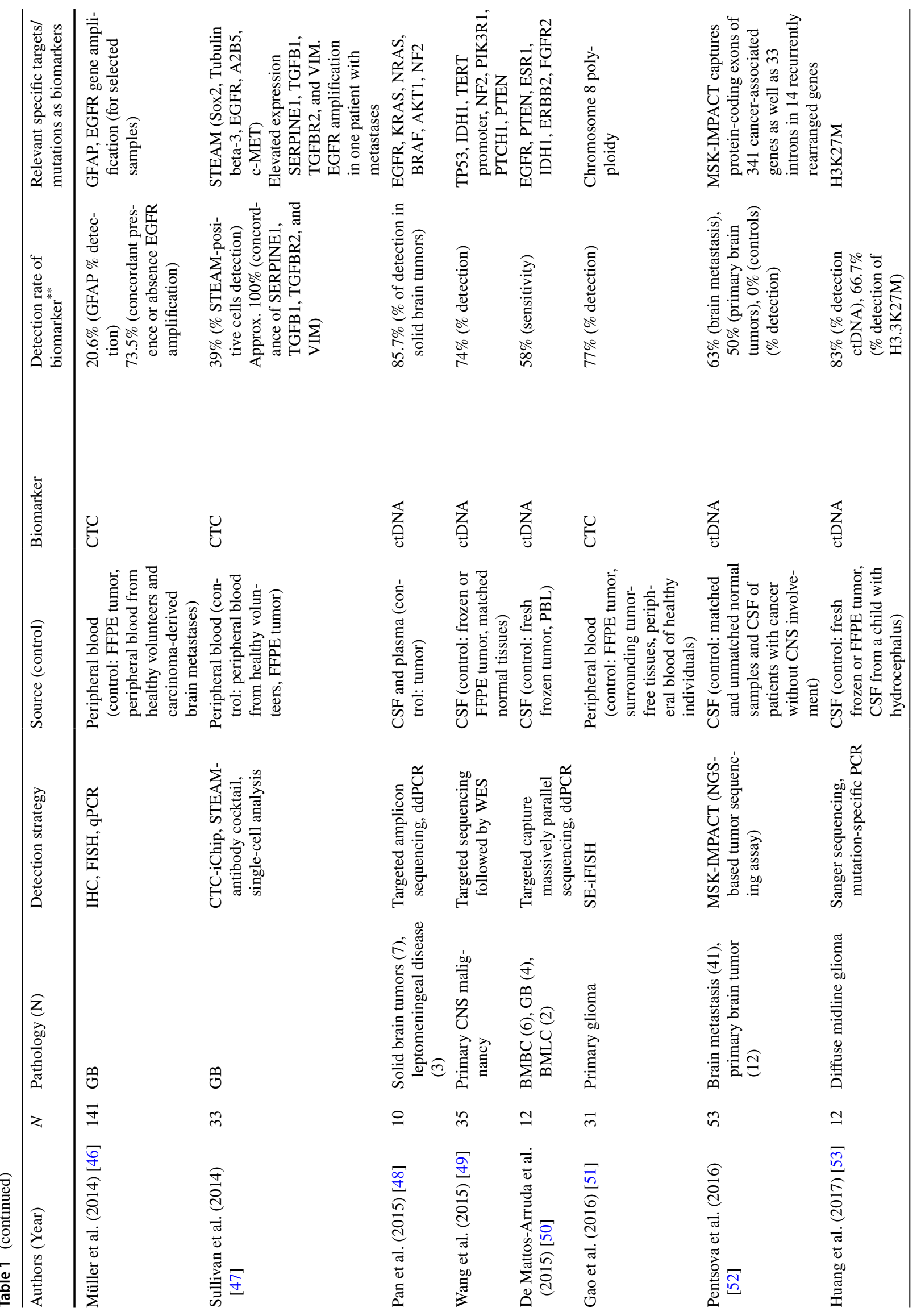




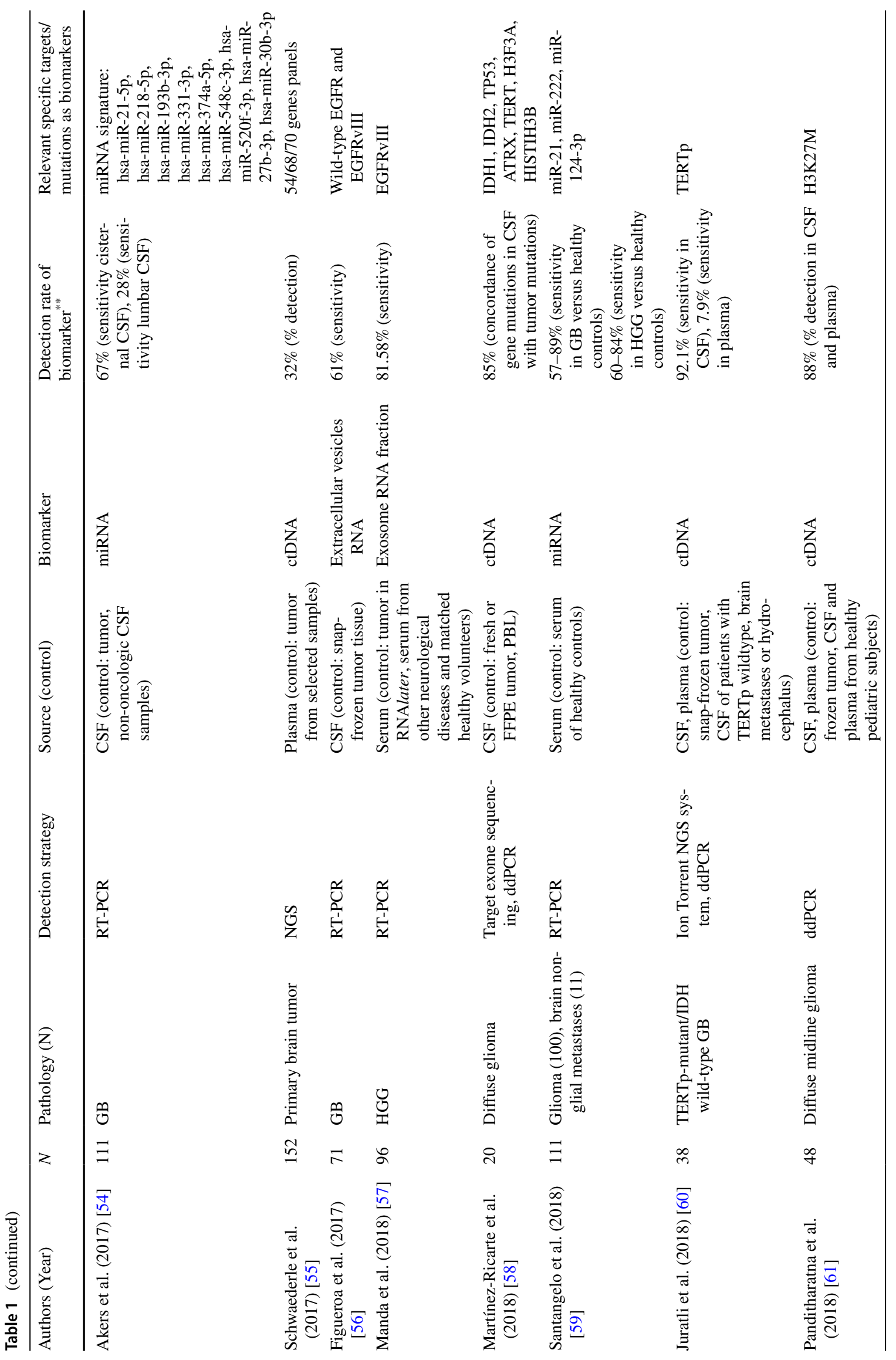




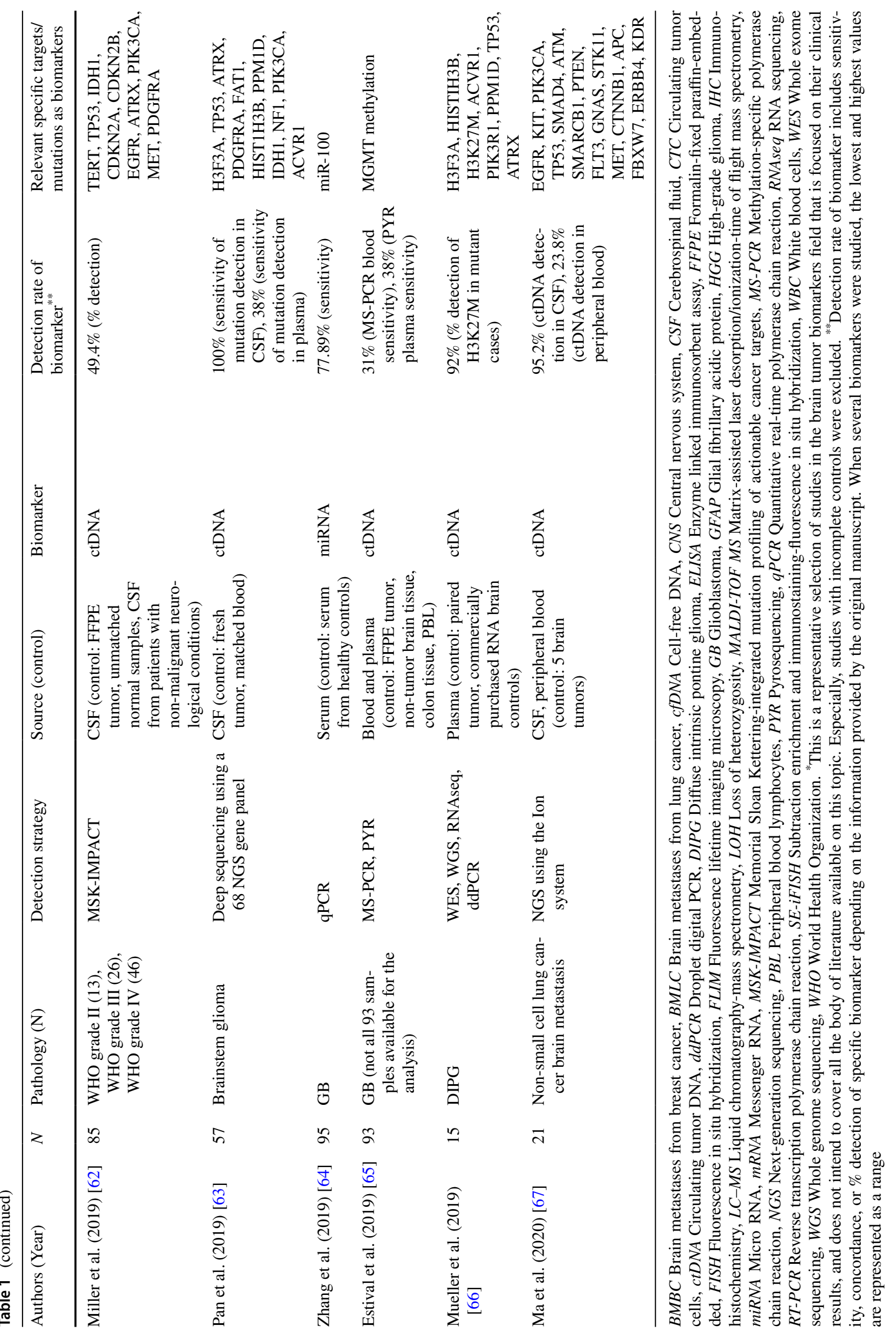


Fig. 1 Representation of Figures I, II and III and extract from the original manuscript from Ashworth TR. A Case of Cancer in Which Cells Similar to Those in the Tumours Were Seen in the Blood after Death. Med J Aust. 1869;14:146-147. A 38-year old man who died of "marasmus" was found to have many subcutaneous nodules. The nodules were made of a jelly-like substance. Fig I and Fig II represent the cells that Ashworth observed in the subcutaneous tumors. Fig III represents Ashworth's observation of cells in the blood that had an appearance similar to those of the subcutaneous tumors, together with other blood "corpuscles" as he describes in the text. This is considered the first description of circulating tumor cells in the English literature. These images are courtesy of the University of Melbourne Archives and are reproduced with their permission and permission from the Medical Journal of Australia

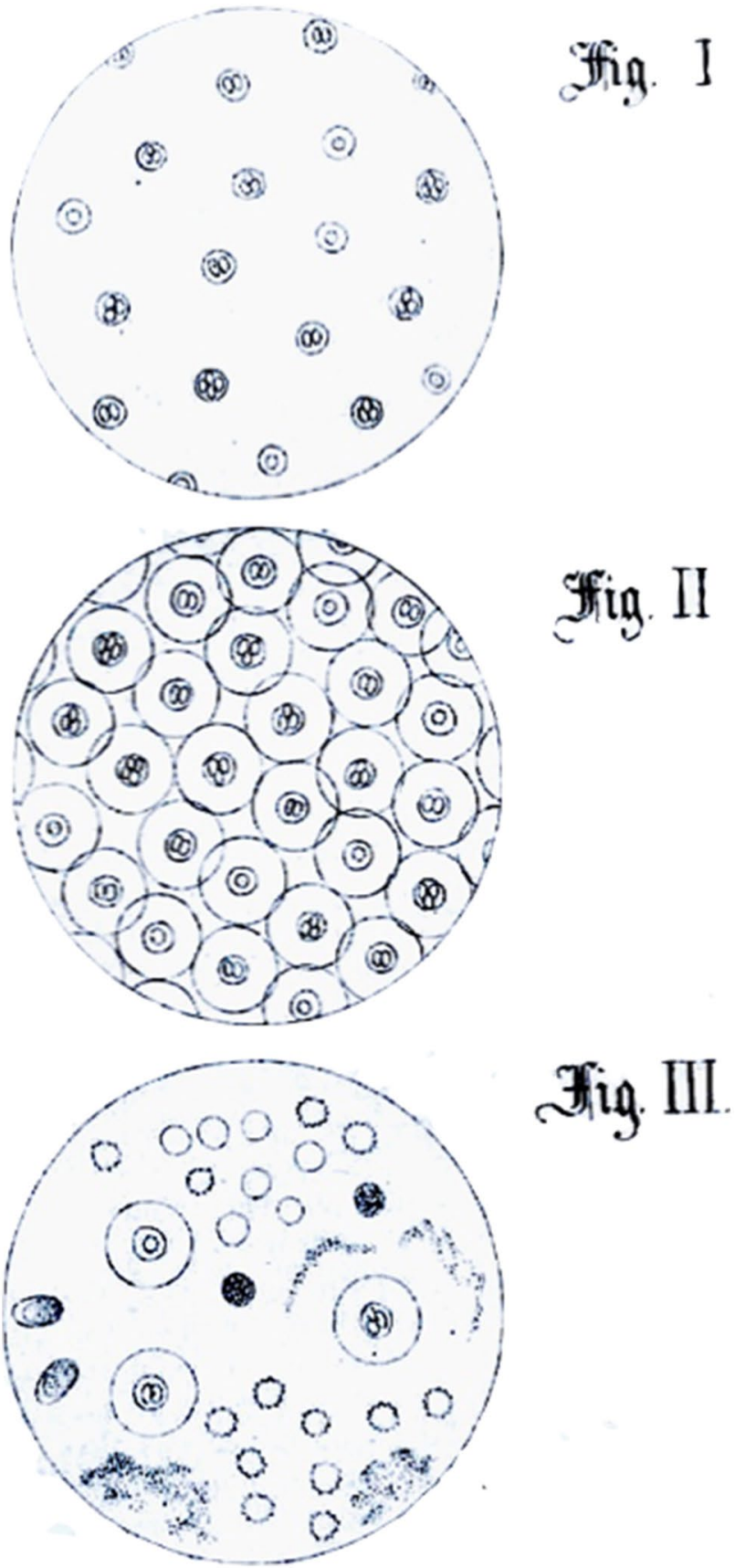

This blood was dark and fluid, when viewed by the microscope a little of the magenta solution being added, it was seen to contain comparatively few red corpuscles, these being mostly shrivelled and stellate, large numbers of white corpuscles, often aggregated; together in masses, besides these some cells like white corpuscles only larger, together with patches of granular matter which took the dye; but the most singular circumstance was that occasionally cells were seen exactly in shape, size, and appearance like to those of the tumours. On one examination three were seen in the field of vision at the same time.-(Fig III.) 
effective way to amplify the detection of these biomarkers in liquid biopsies in a noninvasive, spatially, and temporally controlled manner.

\section{Focused ultrasound and liquid biopsy}

The combination of FUS and biomarkers was initially proposed by D'Souza et al. for human colon cancer lines in 2009 [95]. Many subsequent studies were based on the in vitro and in vivo application of HIFU in non-brain cancer tumors. For example, Chevillet et al. enhanced the release of tumor microRNA in a rat prostate cancer model [96]. D'Souza et al. increased the protein biomarkers in patients with uterine fibroids that underwent ablation by MRgFUS [97]. However, the high intensity of HIFU prevented the early application of this technique to the delicate brain tissue. Lower-intensity pulsed FUS with microbubbles have been used to study FUS and brain tumor biomarkers [78-81]. Table 2 summarizes several relevant manuscripts on FUSenhanced liquid biopsy for brain tumors. This application has been partially explored in murine models. In 2018, Zhu et al. proposed that FUS combined with microbubbles induced disruption of the BBB that allowed both increased import and export of analytes between the brain tissue and the circulation in two orthotopic GB murine models [78]. The investigators implanted orthotopic enhanced green fluorescent protein (eGFP)-transfected human or murine glioma cells (U87 and GL261) and treated them with US-guided FUS or MRgFUS. Quantitative polymerase chain reaction (qPCR) was used to evaluate eGFP mRNA in mice blood four minutes after sonication. Circulating eGFP mRNA was significantly higher in the treated groups compared to the controls. In their proof of concept, the investigators used relatively high acoustic pressures (1.52-3.82 $\mathrm{MPa}$ ), which consequently caused frequent FUS-induced hemorrhage at the tumor sites. Interestingly, there was no difference in biomarker release between different pressures at this higher range. The same team presented a follow-up study in 2020, now evaluating the optimal sonication pressure at which increased biomarker detection was achieved without causing hemorrhage in a murine GB model [79]. Similarly, they implanted orthotopic eGFP-transduced GL261 murine GB cells and treated them with MRgFUS and microbubbles when the tumor reached $2 \mathrm{~mm}$. The researchers compared different peak negative pressure levels, used eGFP mRNA plasma levels as their biomarker of choice, and followed complications with MRI before and after sonication. A 2000- and 8000- fold increase in plasma eGFP mRNA was achieved at the higher pressures (1.29 $\mathrm{MPa}$ and 1.58 MPa). The sonication pressure for optimal biomarker release and low risk of hemorrhage was $0.59 \mathrm{MPa}$, reaching a 55 to 221-fold increase in eGFP mRNA compared to the controls.
In their study, increased tumoral and peritumoral enhancement was associated with increased biomarker release but also post-sonication hemorrhage. To further evaluate their findings in larger animal models, the same group presented a porcine study by Pacia et al. in 2020 [80]. The researchers assessed the BBB opening in healthy pigs with MRgFUS. Increased contrast enhancement in post-sonication MRI and increased $\mathrm{K}_{\text {trans }}$ of gadolinium confirmed the BBB opening. Given the lack of pathology in their porcine model, their selected biomarkers were glial fibrillary acidic protein and myelin basic protein. After sonication, the researchers showed an increased concentration of those brain-specific biomarkers without major clinical or histological brain tissue damage. These summarized preclinical studies in animal models set the foundation for human clinical trials. There may be an optimal sonication pressure in patients that allows biomarker release with a low risk of hemorrhage like in the animal models. Likewise, higher sonication pressures may increase the risk of hemorrhage while the biomarker release plateaus.

\section{Clinical translation for brain tumors}

In 2021, Meng and colleagues presented one of the first published evaluations of cfDNA detection enriched by MRgFUS in patients with pathologically confirmed GB [81]. In their prospective single-arm trial of GB patients, the authors treated nine patients with serial transcranial low-frequency MRgFUS and adjuvant TMZ combination. Their selected biomarkers were plasma cfDNA, neuron-derived extracellular vesicles (NDEV), and brain-specific protein S100b. NCAM and L1CAM surface proteins were used as surrogates for NDEV. Blood samples were collected on average $34 \mathrm{~min}$ after the last sonication. After sonication, the authors showed a 2.6, 3.2, and 1.4-fold increase in cfDNA, NDEV, and $\mathrm{S} 100 \mathrm{~b}$, respectively. As anecdotal evidence, a two-fold to three-fold increase of IDH1-R123H mutant copies was detected by droplet digital PCR in plasma after sonication of the only patient with an IDH mutation. No major complications were reported. Despite the many limitations noted in their manuscript, Meng et al. set the basis for applying MRgFUS-based liquid biopsy in patients with GB.

There are several completed and active clinical trials for the independent study of biomarkers in brain tumors or the safety of MRgFUS. For example, The Mayo Clinic is building a biorepository of CSF samples to analyze biomarkers (NCT04692324). Comparatively, the University of Maryland is assessing the safety and feasibility of ExAblate BBB disruption for treating high-grade gliomas in the context of standard chemotherapy (NCT03322813). 


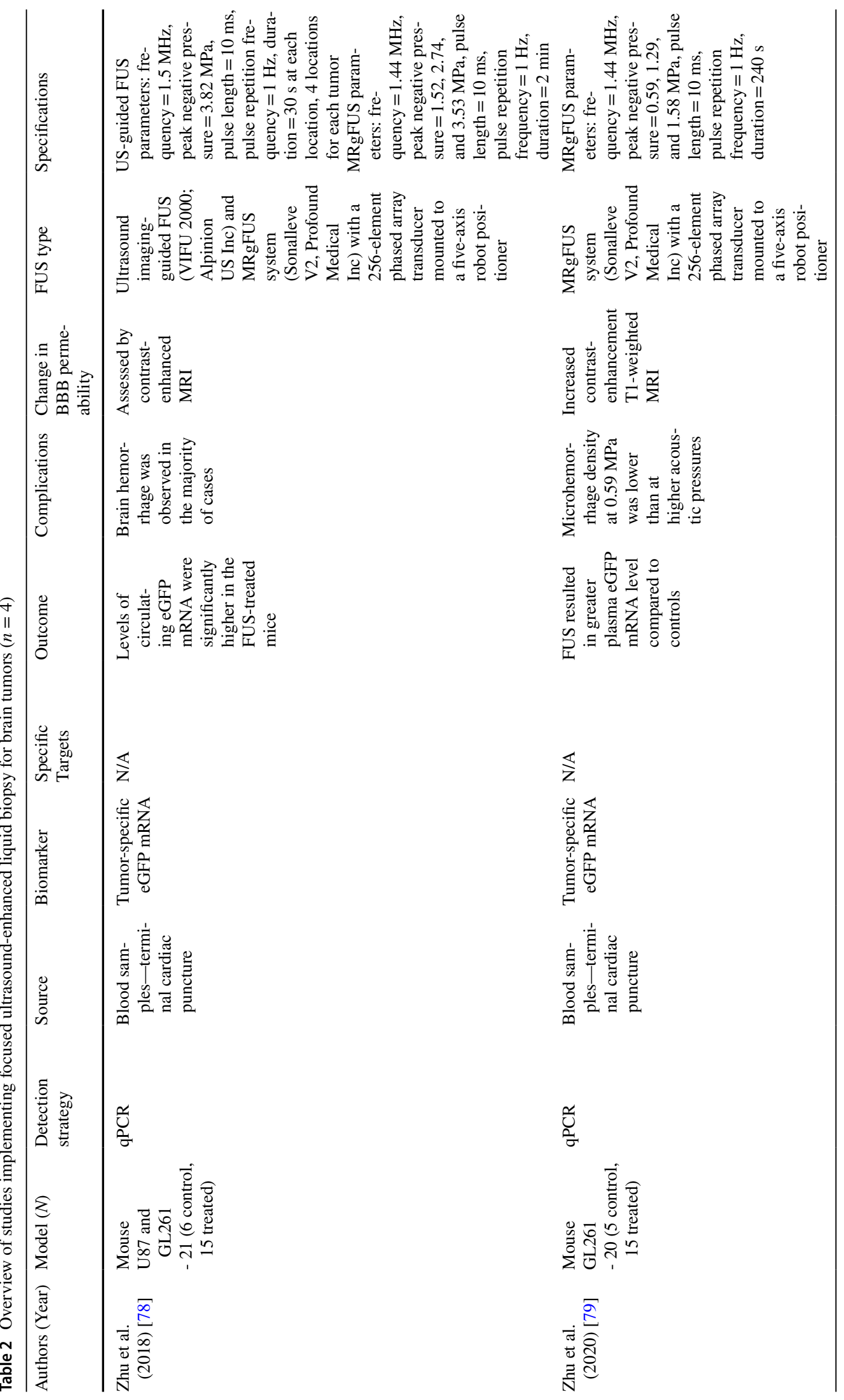




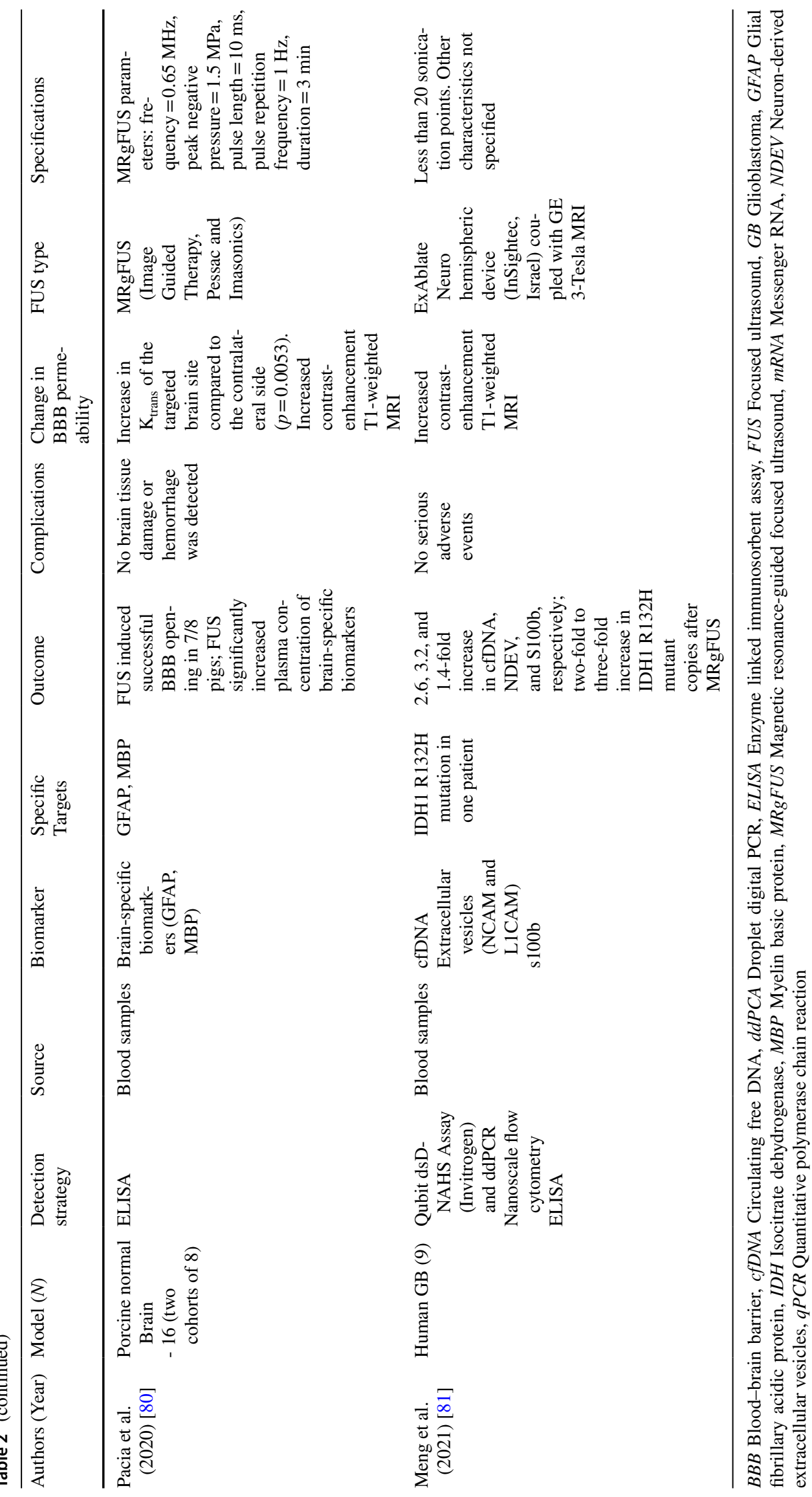


Several other groups are following, and we are awaiting the results of vital clinical trials at the forefront of combining both liquid biopsy and FUS. For example, the BRAINFUL (BRAIN Tumor Focused Ultrasound-enabled Liquid Biopsy) Trial (NCT04940507) is a prospective, singlecenter, single-arm trial that hopes to enhance the detection of ctDNA by MRgFUS in glioma patients while understanding the changes in ctDNA over time. The BRAINFUL Trial, conducted by Dr. Lozano in Toronto, will analyze cfDNA in CSF and blood of patients that undergo tumor ablation with MRgFUS. Additionally, several grants have been recently awarded by the NIH to promote solid advances in this field. Amongst others, research teams at Washington University (1R01EB030102-01), Massachusetts General Hospital (5R01CA239078-02), and our team at Johns Hopkins, in collaboration with the University of Maryland (1R21NS113016-01), are working to revolutionize the detection and monitoring of brain tumors with the development of FUS-enabled liquid biopsies.

\section{Current limitations}

The initiatives mentioned above will establish the foundation to translate FUS-enhanced liquid biopsy to the management of brain tumor patients. Those initiatives aim to overcome several current limitations. As described, available preclinical studies have used artificial biomarkers such as eGFP, which may not fully recapitulate the release of tumor associated biomarkers in the clinical setting. Additionally, terminal blood extractions shortly after sonication in animal experiments prevent the evaluation of FUS-induced biomarker release over time. Further studies are needed to assess the optimal sonication pressures and microbubble parameters for human brain tumor biomarker release while preserving safety. Evaluating biomarker release over time and with the influence of treatments such as radiation and chemotherapy is also paramount to understand the feasibility of FUS-enabled liquid biopsies. Tumor type, density, anatomical location, depth, skull physiology, surrounding vascular structures, and tumor-specific biomarkers may all require different parameters for optimal application of this evolving technique.

The clinical application of liquid biopsies for cancer requires rigorous assay standardization, automatization, and technical and clinical validation to analyze a large volume of samples. At the same time, one of the main limitations for the brain tumor application of MRgFUS is the frailty of the brain parenchyma. Another consideration is that current MRgFUS systems are slow, expensive, and require customization. Given the need for such specialized technology, the economic burden of current detection strategies limits its uptake. With eagerness, the scientific community works to provide a faster, more reliable, and more affordable technology. Several academic and private institutions, such as the European Liquid Biopsy Society or the International Liquid Biopsy Standardization Alliance, coordinated by the Foundation for the NIH, are working to accelerate those much needed technological advances.

Finally, ongoing clinical trials are often centered on advanced or recurrent brain tumors. Still, as knowledge and techniques progress, the goal is to detect early-stage cancers and improve the sensitivity to a point where early detection of recurrences precedes radiographic recurrence and promotes early intervention for improved survival in brain tumor patients.

\section{Conclusion}

Many advances on liquid biopsies and MRgFUS for brain tumors have emerged over the past years. Discovering the possibilities of each independent method allowed us to explore the concept of FUS-enabled liquid biopsy. Understanding both techniques' biological and technological limitations gives us clear goals to overcome over the next decade. Some crucial aims are improving ctDNA detection methods and optimizing FUS settings to maximize biomarker release depending on the pathology and tumor location. Active research endeavors and clinical trials are underway. MRgFUS-enabled liquid biopsy is a revolutionary research field that promises a radical advance in diagnosing and monitoring brain tumors.

Author contributions All the authors in this manuscript made substantial contributions to the conception, design, analysis, interpretation, and creation of this manuscript. All the authors reviewed the final version of the manuscript and agree with its contents and accuracy.

Funding JRT is an NINDS R25 training grant awardee (5R25NS065729). CB is supported by NINDS 1R21NS113016-01.

Data availability The authors confirm that the data supporting the findings of this study are available within the article.

\section{Declarations}

Conflict of interest CB is a consultant for Depuy-Synthes and Bionaut Labs.

Ethical approval This publication is based on the review of the literature and does not report the results of studies involving human subjects and/or animals.

Consent for publication Our manuscript includes a figure and text from the following citation: Ashworth TR. A Case of Cancer in Which Cells Similar to Those in the Tumours Were Seen in the Blood after Death. Med J Aust. 1869;14:146-147. We have requested permission for publication to both, the Medical Journal of Australia, and the Library 
Archives of the University of Melbourne. Both confirmed that under Australian law, the copyright for that publication and all related figures has expired. Corresponding acknowledgements are included in the figure legend and our formal communications with those two entities are included in this submission.

Open Access This article is licensed under a Creative Commons Attribution 4.0 International License, which permits use, sharing, adaptation, distribution and reproduction in any medium or format, as long as you give appropriate credit to the original author(s) and the source, provide a link to the Creative Commons licence, and indicate if changes were made. The images or other third party material in this article are included in the article's Creative Commons licence, unless indicated otherwise in a credit line to the material. If material is not included in the article's Creative Commons licence and your intended use is not permitted by statutory regulation or exceeds the permitted use, you will need to obtain permission directly from the copyright holder. To view a copy of this licence, visit http://creativecommons.org/licenses/by/4.0/.

\section{References}

1. Ostrom QT, Patil N, Cioffi G, Waite K, Kruchko C, BarnholtzSloan JS (2020) CBTRUS statistical report: primary brain and other central nervous system tumors diagnosed in the United States in 2013-2017. Neuro Oncol. https://doi.org/10.1093/neuonc/noaa200

2. Thakkar JP, Dolecek TA, Horbinski C et al (2014) Epidemiologic and molecular prognostic review of glioblastoma. Cancer Epidemiol Biomarkers Prev. https://doi.org/10.1158/1055-9965. EPI-14-0275

3. Stupp R, Mason WP, van den Bent MJ et al (2005) Radiotherapy plus concomitant and adjuvant temozolomide for glioblastoma. $\mathrm{N}$ Engl J Med. https://doi.org/10.1056/NEJMoa043330

4. Almeida JP, Chaichana KL, Rincon-Torroella J, Quinones-Hinojosa A (2015) The value of extent of resection of glioblastomas: clinical evidence and current approach. Curr Neurol Neurosci Rep. https://doi.org/10.1007/s11910-014-0517-x

5. Chaichana KL, Jusue-Torres I, Navarro-Ramirez R et al (2014) Establishing percent resection and residual volume thresholds affecting survival and recurrence for patients with newly diagnosed intracranial glioblastoma. Neuro Oncol. https://doi.org/10. 1093/neuonc/not137

6. Sanai N, Polley M-Y, McDermott MW, Parsa AT, Berger MS (2011) An extent of resection threshold for newly diagnosed glioblastomas. J Neurosurg. https://doi.org/10.3171/2011.2.JNS10998

7. Elshafeey N, Kotrotsou A, Hassan A et al (2019) Multicenter study demonstrates radiomic features derived from magnetic resonance perfusion images identify pseudoprogression in glioblastoma. Nat Commun 10(1):3170. https://doi.org/10.1038/ s41467-019-11007-0

8. Deeken JF, Löscher W (2007) The blood-brain barrier and cancer: transporters, treatment, and trojan horses. Clin Cancer Res. https:// doi.org/10.1158/1078-0432.CCR-06-2854

9. Ballabh P, Braun A, Nedergaard M (2004) The blood-brain barrier: an overview. Neurobiol Dis. https://doi.org/10.1016/j.nbd. 2003.12.016

10. Abbott NJ, Patabendige AAK, Dolman DEM, Yusof SR, Begley DJ (2010) Structure and function of the blood-brain barrier. Neurobiol Dis. https://doi.org/10.1016/j.nbd.2009.07.030

11. Pardridge WM (2005) The blood-brain barrier and neurotherapeutics. NeuroRX. https://doi.org/10.1602/neurorx.2.1.1
12. Oberoi RK, Parrish KE, Sio TT, Mittapalli RK, Elmquist WF, Sarkaria JN (2016) Strategies to improve delivery of anticancer drugs across the blood-brain barrier to treat glioblastoma. Neuro Oncol. https://doi.org/10.1093/neuonc/nov164

13. Ostermann S, Csajka C, Buclin T et al (2004) Plasma and cerebrospinal fluid population pharmacokinetics of temozolomide in malignant glioma patients. Clin cancer Res an Off J Am Assoc Cancer Res 10(11):3728-3736. https://doi.org/10.1158/10780432.CCR-03-0807

14. Lee CY (2017) Strategies of temozolomide in future glioblastoma treatment. Onco Targets Ther. https://doi.org/10.2147/OTT.S1206 62

15. Lonser RR, Sarntinoranont M, Morrison PF, Oldfield EH (2015) Convection-enhanced delivery to the central nervous system. J Neurosurg 122(3):697-706. https://doi.org/10.3171/2014.10. JNS14229

16. Gill SS, Patel NK, Hotton GR et al (2003) Direct brain infusion of glial cell line-derived neurotrophic factor in Parkinson disease. Nat Med. https://doi.org/10.1038/nm850

17. Warren K, Jakacki R, Widemann B et al (2006) Phase II trial of intravenous lobradimil and carboplatin in childhood brain tumors: a report from the Children's Oncology Group. Cancer Chemother Pharmacol 58(3):343-347. https://doi.org/10.1007/ s00280-005-0172-7

18. Ohshima-Hosoyama S, Simmons HA, Goecks N et al (2012) A monoclonal antibody-GDNF fusion protein is not neuroprotective and is associated with proliferative pancreatic lesions in Parkinsonian monkeys. PLoS One. https://doi.org/10.1371/journal.pone. 0039036

19. Wang S, Wu C-C, Zhang H et al (2020) Focused ultrasound induced-blood-brain barrier opening in mouse brain receiving radiosurgery dose of radiation enhances local delivery of systemic therapy. Br J Radiol. https://doi.org/10.1259/bjr.20190214

20. Wei H-J, Upadhyayula PS, Pouliopoulos AN et al (2021) Focused ultrasound-mediated blood-brain barrier opening increases delivery and efficacy of etoposide for glioblastoma treatment. Int J Radiat Oncol. https://doi.org/10.1016/j.ijrobp.2020.12.019

21. Wei K-C, Chu P-C, Wang H-YJ et al (2013) Focused ultrasoundinduced blood-brain barrier opening to enhance temozolomide delivery for glioblastoma treatment: a preclinical study. PLoS One. https://doi.org/10.1371/journal.pone.0058995

22. Treat LH, McDannold N, Zhang Y, Vykhodtseva N, Hynynen K (2012) Improved anti-tumor effect of liposomal doxorubicin after targeted blood-brain barrier disruption by mri-guided focused ultrasound in rat glioma. Ultrasound Med Biol. https://doi.org/ 10.1016/j.ultrasmedbio.2012.04.015

23. Aryal M, Vykhodtseva N, Zhang Y-Z, Park J, McDannold N (2013) Multiple treatments with liposomal doxorubicin and ultrasound-induced disruption of blood-tumor and blood-brain barriers improve outcomes in a rat glioma model. J Control Release. https://doi.org/10.1016/j.jconrel.2013.04.007

24. Kovacs Z, Werner B, Rassi A, Sass JO, Martin-Fiori E, Bernasconi M (2014) Prolonged survival upon ultrasound-enhanced doxorubicin delivery in two syngenic glioblastoma mouse models. J Control Release. https://doi.org/10.1016/j.jconrel.2014.05.033

25. Chen P-Y, Hsieh H-Y, Huang C-Y, Lin C-Y, Wei K-C, Liu H-L (2015) Focused ultrasound-induced blood-brain barrier opening to enhance interleukin-12 delivery for brain tumor immunotherapy: a preclinical feasibility study. J Transl Med. https://doi.org/ 10.1186/s12967-015-0451-y

26. Alkins R, Burgess A, Ganguly M et al (2013) Focused ultrasound delivers targeted immune cells to metastatic brain tumors. Cancer Res. https://doi.org/10.1158/0008-5472.CAN-12-2609

27. Burgess A, Ayala-Grosso CA, Ganguly M, Jordão JF, Aubert I, Hynynen K (2011) Targeted delivery of neural stem cells to the brain using MRI-guided focused ultrasound to disrupt the 
blood-brain barrier. PLoS One. https://doi.org/10.1371/journal. pone. 0027877

28. Liu H-L, Hsu P-H, Lin C-Y et al (2016) Focused ultrasound enhances central nervous system delivery of bevacizumab for malignant glioma treatment. Radiology. https://doi.org/10.1148/ radiol.2016152444

29. Papachristodoulou A, Signorell RD, Werner B et al (2019) Chemotherapy sensitization of glioblastoma by focused ultrasound-mediated delivery of therapeutic liposomes. J Control Release. https://doi.org/10.1016/j.jconrel.2018.12.009

30. Liu H-L, Hua M-Y, Chen P-Y et al (2010) Blood-brain barrier disruption with focused ultrasound enhances delivery of chemotherapeutic drugs for glioblastoma treatment. Radiology. https:// doi.org/10.1148/radiol.10090699

31. Cescon DW, Bratman SV, Chan SM, Siu LL (2020) Circulating tumor DNA and liquid biopsy in oncology. Nat Cancer. https:// doi.org/10.1038/s43018-020-0043-5

32. Louis DN, Perry A, Wesseling P et al (2021) The 2021 WHO classification of tumors of the central nervous system: a summary. Neuro Oncol. https://doi.org/10.1093/neuonc/noab106

33. Malone H, Yang J, Hershman DL, Wright JD, Bruce JN, Neugut AI (2015) Complications following stereotactic needle biopsy of intracranial tumors. World Neurosurg. https://doi.org/10.1016/j. wneu.2015.05.025

34. Kickingereder P, Willeit P, Simon T, Ruge MI (2013) Diagnostic value and safety of stereotactic biopsy for brainstem tumors: a systematic review and meta-analysis of 1480 cases. Neurosurgery 72(6):873-881. https://doi.org/10.1227/NEU.0b013e3182 8 bf445 (discussion 882; quiz 882)

35. Mattox AK, Yan H, Bettegowda C (2019) The potential of cerebrospinal fluid-based liquid biopsy approaches in CNS tumors. Neuro Oncol 21(12):1509-1518. https://doi.org/10.1093/neuonc/noz156

36. Wang J, Bettegowda C (2017) Applications of DNA-based liquid biopsy for central nervous system neoplasms. J Mol Diagn 19(1):24-34. https://doi.org/10.1016/j.jmoldx.2016.08.007

37. Balaña C, Ramirez JL, Taron M et al (2003) O6-methyl-guanine-DNA methyltransferase methylation in serum and tumor DNA predicts response to 1,3-bis(2-chloroethyl)-1-nitrosourea but not to temozolamide plus cisplatin in glioblastoma multiforme. Clin Cancer Res 9(4):1461-1468

38. Weaver KD, Grossman SA, Herman JG (2006) Methylated tumor-specific DNA as a plasma biomarker in patients with glioma. Cancer Invest 24(1):35-40. https://doi.org/10.1080/ 07357900500449546

39. Skog J, Würdinger T, van Rijn S et al (2008) Glioblastoma microvesicles transport RNA and proteins that promote tumour growth and provide diagnostic biomarkers. Nat Cell Biol 10(12):1470-1476. https://doi.org/10.1038/ncb1800

40. Lavon I, Refael M, Zelikovitch B, Shalom E, Siegal T (2010) Serum DNA can define tumor-specific genetic and epigenetic markers in gliomas of various grades. Neuro Oncol 12(2):173180. https://doi.org/10.1093/neuonc/nop041

41. Saratsis AM, Yadavilli S, Magge S et al (2012) Insights into pediatric diffuse intrinsic pontine glioma through proteomic analysis of cerebrospinal fluid. Neuro Oncol 14(5):547-560. https://doi.org/10.1093/neuonc/nos067

42. Boisselier B, Gállego Pérez-Larraya J, Rossetto M et al (2012) Detection of IDH1 mutation in the plasma of patients with glioma. Neurology 79(16):1693-1698. https://doi.org/10.1212/ WNL.0b013e31826e9b0a

43. Majchrzak-Celińska A, Paluszczak J, Kleszcz R et al (2013) Detection of MGMT, RASSF1A, p15INK4B, and p14ARF promoter methylation in circulating tumor-derived DNA of central nervous system cancer patients. J Appl Genet 54(3):335-344. https://doi.org/10.1007/s13353-013-0149-x
44. Bettegowda C, Sausen M, Leary RJ et al (2014) Detection of circulating tumor DNA in early- and late-stage human malignancies. Sci Transl Med. https://doi.org/10.1126/scitranslmed. 3007094

45. Macarthur KM, Kao GD, Chandrasekaran S et al (2014) Detection of brain tumor cells in the peripheral blood by a telomerase promoter-based assay. Cancer Res 74(8):2152-2159. https://doi. org/10.1158/0008-5472.CAN-13-0813

46. Müller C, Holtschmidt J, Auer M et al (2014) Hematogenous dissemination of glioblastoma multiforme. Sci Transl Med. https://doi.org/10.1126/scitranslmed.3009095

47. Sullivan JP, Nahed BV, Madden MW et al (2014) Brain tumor cells in circulation are enriched for mesenchymal gene expression. Cancer Discov 4(11):1299-1309. https://doi.org/10.1158/ 2159-8290.CD-14-0471

48. Pan W, Gu W, Nagpal S, Gephart MH, Quake SR (2015) Brain tumor mutations detected in cerebral spinal fluid. Clin Chem 61(3):514-522. https://doi.org/10.1373/clinchem.2014.235457

49. Wang Y, Springer S, Zhang M et al (2015) Detection of tumorderived DNA in cerebrospinal fluid of patients with primary tumors of the brain and spinal cord. Proc Natl Acad Sci U S A 112(31):9704-9709. https://doi.org/10.1073/pnas.1511694112

50. De Mattos-Arruda L, Mayor R, Ng CKY et al (2015) Cerebrospinal fluid-derived circulating tumour DNA better represents the genomic alterations of brain tumours than plasma. Nat Commun 6:8839. https://doi.org/10.1038/ncomms9839

51. Gao F, Cui Y, Jiang H et al (2016) Circulating tumor cell is a common property of brain glioma and promotes the monitoring system. Oncotarget 7(44):71330-71340. https://doi.org/10. 18632/oncotarget.11114

52. Pentsova EI, Shah RH, Tang J et al (2016) Evaluating cancer of the central nervous system through next-generation sequencing of cerebrospinal fluid. J Clin Oncol Off J Am Soc Clin Oncol 34(20):2404-2415. https://doi.org/10.1200/JCO.2016.66.6487

53. Huang TY, Piunti A, Lulla RR et al (2017) Detection of Histone H3 mutations in cerebrospinal fluid-derived tumor DNA from children with diffuse midline glioma. Acta Neuropathol Commun 5(1):28. https://doi.org/10.1186/s40478-017-0436-6

54. Akers JC, Hua W, Li H et al (2017) A cerebrospinal fluid microRNA signature as biomarker for glioblastoma. Oncotarget 8(40):68769-68779. https://doi.org/10.18632/oncotarget.18332

55. Schwaederle M, Chattopadhyay R, Kato S et al (2017) Genomic alterations in circulating tumor DNA from diverse cancer patients identified by next-generation sequencing. Cancer Res 77(19):5419-5427. https://doi.org/10.1158/0008-5472. CAN-17-0885

56. Figueroa JM, Skog J, Akers J et al (2017) Detection of wild-type EGFR amplification and EGFRvIII mutation in CSF-derived extracellular vesicles of glioblastoma patients. Neuro Oncol 19(11):1494-1502. https://doi.org/10.1093/neuonc/nox085

57. Manda SV, Kataria Y, Tatireddy BR et al (2018) Exosomes as a biomarker platform for detecting epidermal growth factor receptor-positive high-grade gliomas. J Neurosurg 128(4):10911101. https://doi.org/10.3171/2016.11.JNS161187

58. Martínez-Ricarte F, Mayor R, Martínez-Sáez E et al (2018) Molecular diagnosis of diffuse gliomas through sequencing of cell-free circulating tumor DNA from cerebrospinal fluid. Clin cancer Res an Off J Am Assoc Cancer Res 24(12):2812-2819. https://doi.org/10.1158/1078-0432.CCR-17-3800

59. Santangelo A, Imbrucè P, Gardenghi B et al (2018) A microRNA signature from serum exosomes of patients with glioma as complementary diagnostic biomarker. J Neurooncol 136(1):5162. https://doi.org/10.1007/s11060-017-2639-x

60. Juratli TA, Stasik S, Zolal A et al (2018) TERT promoter mutation detection in cell-free tumor-derived DNA in patients with IDH wild-type glioblastomas: A pilot prospective study. Clin 
cancer Res an Off J Am Assoc Cancer Res 24(21):5282-5291. https://doi.org/10.1158/1078-0432.CCR-17-3717

61. Panditharatna E, Kilburn LB, Aboian MS et al (2018) Clinically relevant and minimally invasive tumor surveillance of pediatric diffuse midline gliomas using patient-derived liquid biopsy. Clin cancer Res an Off J Am Assoc Cancer Res 24(23):5850 5859. https://doi.org/10.1158/1078-0432.CCR-18-1345

62. Miller AM, Shah RH, Pentsova EI et al (2019) Tracking tumour evolution in glioma through liquid biopsies of cerebrospinal fluid. Nature 565(7741):654-658. https://doi.org/10.1038/ s41586-019-0882-3

63. Pan C, Diplas BH, Chen X et al (2019) Molecular profiling of tumors of the brainstem by sequencing of CSF-derived circulating tumor DNA. Acta Neuropathol 137(2):297-306. https://doi. org/10.1007/s00401-018-1936-6

64. Zhang H, Wang J, Wang Z, Ruan C, Wang L, Guo H (2019) Serum miR-100 is a potential biomarker for detection and outcome prediction of glioblastoma patients. Cancer Biomark 24(1):43-49. https://doi.org/10.3233/CBM-181416

65. Estival A, Sanz C, Ramirez J-L et al (2019) Pyrosequencing versus methylation-specific PCR for assessment of MGMT methylation in tumor and blood samples of glioblastoma patients. Sci Rep 9(1):11125. https://doi.org/10.1038/s41598-019-47642-2

66. Mueller S, Jain P, Liang WS et al (2019) A pilot precision medicine trial for children with diffuse intrinsic pontine gliomaPNOC003: a report from the Pacific pediatric neuro-oncology consortium. Int J cancer 145(7):1889-1901. https://doi.org/10. $1002 /$ ijc. 32258

67. Ma C, Yang X, Xing W, Yu H, Si T, Guo Z (2020) Detection of circulating tumor DNA from non-small cell lung cancer brain metastasis in cerebrospinal fluid samples. Thorac cancer 11(3):588-593. https://doi.org/10.1111/1759-7714.13300

68. Le Rhun E, Seoane J, Salzet M, Soffietti R, Weller M (2020) Liquid biopsies for diagnosing and monitoring primary tumors of the central nervous system. Cancer Lett 480:24-28. https:// doi.org/10.1016/j.canlet.2020.03.021

69. Ashworth TR (1869) A case of cancer in which cells similar to those in the tumours were seen in the blood after death. Med J Aust 14:146-147

70. Mandel P, Métais P (1948) Les acides nucleiques du plasma sanguin chez l' homme. C R Seances Soc Biol Fil 142:241-243

71. Sorenson GD, Pribish DM, Valone FH, Memoli VA, Bzik DJ, Yao SL (1994) Soluble normal and mutated DNA sequences from single-copy genes in human blood. Cancer Epidemiol biomarkers Prev a Publ Am Assoc Cancer Res cosponsored by Am Soc Prev Oncol 3(1):67-71

72. Vasioukhin V, Anker P, Maurice P, Lyautey J, Lederrey C, Stroun M (1994) Point mutations of the N-ras gene in the blood plasma DNA of patients with myelodysplastic syndrome or acute myelogenous leukaemia. Br J Haematol 86(4):774-779. https://doi.org/10.1111/j.1365-2141.1994.tb04828.x

73. Diehl F, Schmidt K, Choti MA et al (2008) Circulating mutant DNA to assess tumor dynamics. Nat Med 14(9):985-990. https://doi.org/10.1038/nm.1789

74. Diehl F, Li M, Dressman D et al (2005) Detection and quantification of mutations in the plasma of patients with colorectal tumors. Proc Natl Acad Sci U S A 102(45):16368-16373. https://doi.org/10.1073/pnas.0507904102

75. Karachaliou N, Mayo-de las Casas C, Queralt C et al (2015) Association of EGFR L858R mutation in circulating free DNA with survival in the EURTAC trial. JAMA Oncol 1(2):149-157. https://doi.org/10.1001/jamaoncol.2014.257

76. Magbanua MJM, Swigart LB, Wu H-T et al (2021) Circulating tumor DNA in neoadjuvant-treated breast cancer reflects response and survival. Ann Oncol Off J Eur Soc Med Oncol 32(2):229-239. https://doi.org/10.1016/j.annonc.2020.11.007
77. Arvanitis CD, Ferraro GB, Jain RK (2020) The blood-brain barrier and blood-tumour barrier in brain tumours and metastases. Nat Rev Cancer. https://doi.org/10.1038/s41568-019-0205-x

78. Zhu L, Cheng G, Ye D et al (2018) Focused ultrasound-enabled brain tumor liquid biopsy. Sci Rep. https://doi.org/10.1038/ s41598-018-24516-7

79. Zhu L, Nazeri A, Pacia CP, Yue Y, Chen H (2020) Focused ultrasound for safe and effective release of brain tumor biomarkers into the peripheral circulation. PLoS One. https://doi.org/10.1371/ journal.pone.0234182

80. Pacia CP, Zhu L, Yang Y et al (2020) Feasibility and safety of focused ultrasound-enabled liquid biopsy in the brain of a porcine model. Sci Rep. https://doi.org/10.1038/s41598-020-64440-3

81. Meng Y, Pople CB, Suppiah S et al (2021) MR-guided focused ultrasound liquid biopsy enriches circulating biomarkers in patients with brain tumors. Neuro Oncol. https://doi.org/10.1093/ neuonc/noab057

82. McDannold N, Vykhodtseva N, Hynynen K (2006) Targeted disruption of the blood-brain barrier with focused ultrasound: association with cavitation activity. Phys Med Biol. https://doi. org/10.1088/0031-9155/51/4/003

83. Ram Z, Cohen ZR, Harnof S et al (2006) Magnetic resonance imaging-guided, high-intensity focused ultrasound for brain tumor therapy. Neurosurgery 59(5):946-949. https://doi.org/10.1227/01. NEU.0000254439.02736.D8

84. Ghanouni P, Pauly KB, Elias WJ et al (2015) Transcranial MRIguided focused ultrasound: a review of the technologic and neurologic applications. Am J Roentgenol. https://doi.org/10.2214/ AJR.14.13632

85. Moser D, Zadicario E, Schiff G, Jeanmonod D (2013) MRguided focused ultrasound technique in functional neurosurgery: targeting accuracy. J Ther Ultrasound. https://doi.org/10.1186/ 2050-5736-1-3

86. Mitragotri S (2005) Healing sound: the use of ultrasound in drug delivery and other therapeutic applications. Nat Rev Drug Discov. https://doi.org/10.1038/nrd1662

87. Sheikov N, McDannold N, Vykhodtseva N, Jolesz F, Hynynen K (2004) Cellular mechanisms of the blood-brain barrier opening induced by ultrasound in presence of microbubbles. Ultrasound Med Biol. https://doi.org/10.1016/j.ultrasmedbio.2004.04.010

88. Meng Y, Suppiah S, Surendrakumar S, Bigioni L, Lipsman N (2018) Low-intensity MR-guided focused ultrasound mediated disruption of the blood-brain barrier for intracranial metastatic diseases. Front Oncol. https://doi.org/10.3389/fonc.2018.00338

89. Leinenga G, Götz J (2015) Scanning ultrasound removes amyloid- $\beta$ and restores memory in an Alzheimer's disease mouse model. Sci Transl Med. https://doi.org/10.1126/scitranslmed. aaa2512

90. Karakatsani ME, Wang S, Samiotaki G et al (2019) Amelioration of the nigrostriatal pathway facilitated by ultrasound-mediated neurotrophic delivery in early Parkinson's disease. J Control Release. https://doi.org/10.1016/j.jconrel.2019.03.030

91. Lipsman N, Meng Y, Bethune AJ et al (2018) Blood-brain barrier opening in Alzheimer's disease using MR-guided focused ultrasound. Nat Commun. https://doi.org/10.1038/s41467-018-04529-6

92. Mainprize T, Lipsman N, Huang Y et al (2019) Blood-Brain barrier opening in primary brain tumors with non-invasive MRguided focused ultrasound: a clinical safety and feasibility study. Sci Rep. https://doi.org/10.1038/s41598-018-36340-0

93. Abrahao A, Meng Y, Llinas M et al (2019) First-in-human trial of blood-brain barrier opening in amyotrophic lateral sclerosis using MR-guided focused ultrasound. Nat Commun. https://doi.org/10. 1038/s41467-019-12426-9 
94. Park SH, Kim MJ, Jung HH et al (2021) Safety and feasibility of multiple blood-brain barrier disruptions for the treatment of glioblastoma in patients undergoing standard adjuvant chemotherapy. J Neurosurg. https://doi.org/10.3171/2019.10.JNS192206

95. D'Souza AL, Tseng JR, Pauly KB et al (2009) A strategy for blood biomarker amplification and localization using ultrasound. Proc Natl Acad Sci U S A 106(40):17152-17157. https://doi.org/10. 1073/pnas.0903437106

96. Chevillet JR, Khokhlova TD, Giraldez MD et al (2017) Molecular imaging: release of cell-free MicroRNA tumor biomarkers into the blood circulation. Radiology 283:158-167
97. D'Souza AL, Chevillet JR, Ghanouni P, Yan X, Tewari M, Gambhir SS (2018) Tumor characterization by ultrasound-release of multiple protein and microRNA biomarkers, preclinical and clinical evidence. PLoS One 13(3):1-17. https://doi.org/10.1371/journ al.pone. 0194268

Publisher's Note Springer Nature remains neutral with regard to jurisdictional claims in published maps and institutional affiliations. 